\title{
Dual-Polarized Patch Antenna-in-Package with High Isolation for Ka-Band 5G Communications
}

\author{
H. M. Santos \\ Faculdade de Engenharia da Universidade do Porto \\ INESC TEC \\ Porto, Portugal \\ hugo.m.santos@inesctec.pt
}

\author{
H. M. Salgado \\ Faculdade de Engenharia da Universidade do Porto \\ INESC TEC \\ Porto, Portugal \\ hsalgado@fe.up.pt
}

\begin{abstract}
In this paper we describe the design of a dual polarized packaged patch antenna for $5 \mathrm{G}$ communications with improved isolation and bandwidth for Ka-band. The results were validated using FEM and Momentum co-simulations in ADS. The novelty of the approach is the use of parasitic elements in the same layer to circumvent bandwidth limitations, thereby reducing the layer count in contrast to previous designs, combined with a differential feeding technique for improved isolation and radiation pattern stability, albeit at the expense of an increased complexity in the matching process. A peak gain of $5 \mathrm{dBi}$, isolation above $40 \mathrm{~dB}$ and a radiation efficiency of $60 \%$ were obtained.
\end{abstract}

Index Terms-Antenna-in-Package, Patch Antenna, Polarization Diversity, 5G

\section{INTRODUCTION}

The european $5 \mathrm{G}$ communication band in the frequency range from 24.25 to $27.5 \mathrm{GHz}$ is settled as the most adequate solution for limited coverage and high throughput scenarios [1]. Its usage drives the need for new antenna technologies capable of beamforming, polarization diversity and large bandwidths.

Patch antennas are a possible solution for the challenge of 5G Ka-band antennas, given their size which facilitates package integration, simplicity and ability to support two polarizations in two degenerate modes. Nonetheless, their bandwidth is usually very limited and bandwidth improvement techniques are generally required in broadband communication systems [2].

The most common solution found in the state-of-the-art to overcome bandwidth limitations is the usage of a stacked patch topology. However, such technique can lead to higher order modes which will degrade radiation pattern stability throughout the band of operation [3]. Furthermore these higher order modes also compromise isolation in dually-polarized

978-1-7281-3099-6/19/\$31.00 @2019 IEEE

\author{
Pedro Pinho \\ Instituto de Telecomunicações \\ ADEETC, Instituto Superior de Engenharia de Lisboa \\ Aveiro, Portugal \\ ppinho@deetc.isel.pt
}

antennas. In some cases, a different patch topology had to be used to improve isolation between polarizations.

In this work we propose the usage of a patch antenna with parasitic elements in the same layer to improve its bandwidth, reducing the layer count from typical stacked patches as reported in [4]. Based on [5], we utilized a differential feeding technique. By doing this, each mode is excited with two feeds with opposing phases, which cancel out higher order modes, reduce feed radiation and reinforce the fundamental degenerate modes $\mathrm{TM}_{010}$ and $\mathrm{TM}_{100}$ of the patch antenna. Ultimately, this improves isolation between polarizations and radiation pattern stability.

\section{Antenna Design}

In this work a land-side die antenna-in-package was considered as shown in the stackup of Fig. 1. The core material is Rogers RO4350B and the prepreg is Rogers RO4450T. These substrates offer a good compromise between cost, moderate permittivity values $\left(\epsilon_{r} \approx 3.5\right)$ and low losses $(\tan \delta \approx 0.004)$. The metal layers are all composed of $17.5 \mu \mathrm{m}$ thick copper.

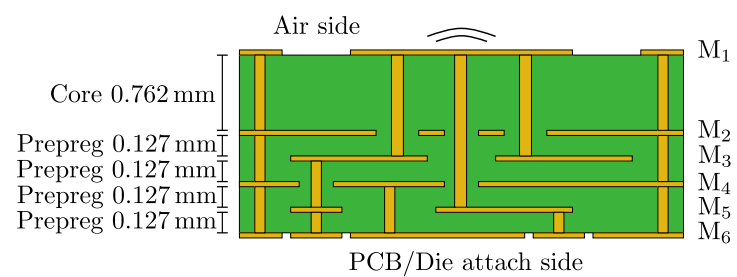

Fig. 1. Antenna stackup.

The patch and its parasitics are designed in $\mathrm{M}_{1}$ metal layer, as shown in Fig. 2. The vertically polarized stream (port 1) is fed from $\mathrm{M}_{3}$ and the horizontal (port 2) from $\mathrm{M}_{5}$ metal layer. In each feeding network a half-wavelength $50 \Omega$ transmission line was connected between the two corresponding balanced ports to obtain the needed $180^{\circ}$ phase shift. Another $50 \Omega$ line was connected to one of the balanced ports in each 
polarization, to evaluate the single-ended reflection coefficient. As seen in Fig. 3, a low reflection coefficient, at the centre frequency of $26 \mathrm{GHz}$, was obtained at both ports, after Momentum simulation in ADS (Advanced Design System), by setting $p s=2.75 \mathrm{~mm}, f o=0.8 \mathrm{~mm}, p l=1.7 \mathrm{~mm}$, $p w=1.2 \mathrm{~mm}, p g=0.1 \mathrm{~mm}$. However a limited bandwidth of approximately $2.5 \mathrm{GHz}$ was verified, which falls short from the needed $3.25 \mathrm{GHz}$.

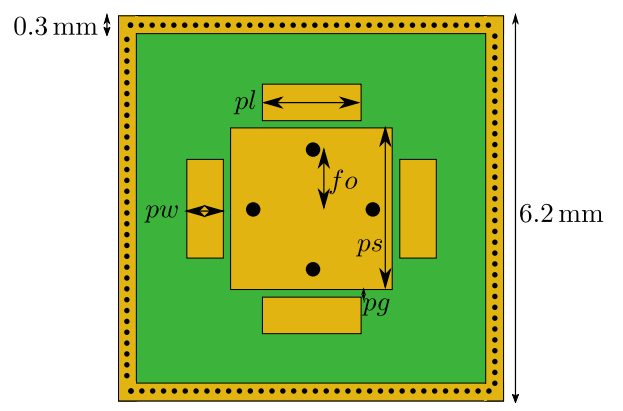

Fig. 2. Antenna layout.

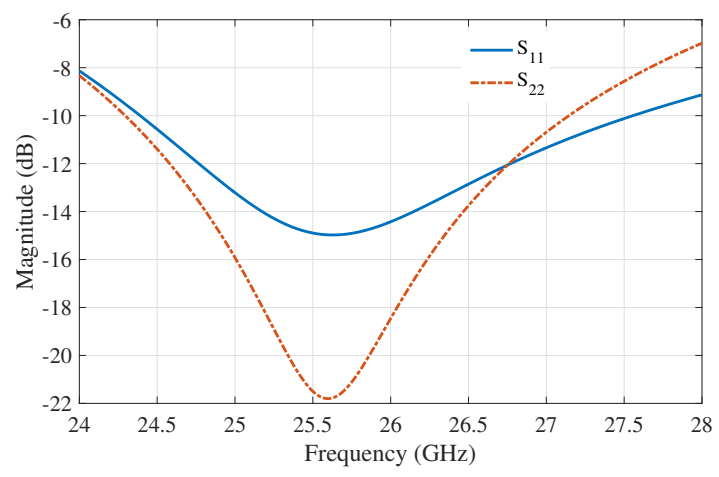

Fig. 3. $S_{11}$ and $S_{22}$ magnitudes as a function of frequency for the patch antenna without matching network.

Because of the bandwidth limitation a matching network had to be designed. Since the transition from the antenna feeding networks to the PCB would present some parasitics, the matching network was designed after such transition was characterized. The full system will be presented in section IV.

\section{PCB to PACKAGE TRAnsition}

The transition between the hosting PCB and the antennain-package was designed as a simple connection between a microstrip on the host $\mathrm{PCB}$ and a stripline on $\mathrm{M}_{5}$ layer of the package. To connect to the feeding network on $\mathrm{M}_{3}$ an internal transition between $\mathrm{M}_{5}$ and $\mathrm{M}_{3}$ was also designed. After FEM simulation return losses higher than $14 \mathrm{~dB}$ and insertion losses lower than $0.25 \mathrm{~dB}$ were obtained which validate the transition.

\section{FinAl Design Evaluation}

To compensate the PCB to package transition parasitics and the reduced bandwidth verified in section II, the procedure was to detune the patch antenna by setting the dimensions $p s=2.83 \mathrm{~mm}$, fo $=0.8 \mathrm{~mm}, p l=1.7 \mathrm{~mm}, p w=0.7 \mathrm{~mm}$, $p g=0.1 \mathrm{~mm}$, so that optimum gain was achieved. A matching network was then introduced to obtain $50 \Omega$ input impedance.

The matching network circuit is shown in Fig. 4 and its values are given in Table I for both the V-Pol and H-Pol inputs of the antenna. Despite the rotational symmetry of the antenna, the matching networks are different because of an added transition between $\mathrm{M}_{3}$ and $\mathrm{M}_{5}$ metal layers, which results in a non-symmetric $\mathrm{S}$-parameter matrix. This transition serves as a connection between $\mathrm{M}_{3}$ layer and the package to PCB transition. The values of both matching networks were obtained by resorting to a PSO (Particle Swarm Optimization) algorithm. The upper bound for the impedance of each transmission line was obtained by calculating the characteristic impedance of a stripline with the minimum trace width of $100 \mu \mathrm{m}$, for easier fabrication. This impedance was calculated using ADS and the value obtained was $Z_{\max }=53.8 \Omega$.

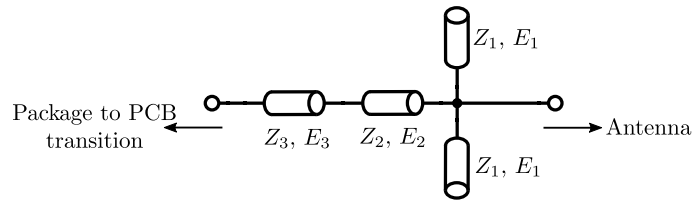

Fig. 4. Matching network schematic.

TABLE I

MATCHING NETWORK PARAMETERS AT THE CENTRE FREQUENCY OF $26 \mathrm{GHz}$.

\begin{tabular}{ccccccc}
\hline Polarization & $Z_{1}$ & $E_{1}$ & $Z_{2}$ & $E_{2}$ & $Z_{3}$ & $E_{3}$ \\
\hline V-Pol & $53.8 \Omega$ & $50^{\circ}$ & $53.8 \Omega$ & $0^{\circ}$ & $20.1 \Omega$ & $107^{\circ}$ \\
H-Pol & $53.8 \Omega$ & $58^{\circ}$ & $53.8 \Omega$ & $26.8^{\circ}$ & $16 \Omega$ & $43.4^{\circ}$
\end{tabular}

The final layout is presented in Fig. 5, where the values of Table I were mapped to striplines. In this layout, the transition between metal layers $\mathrm{M}_{3}$ and $\mathrm{M}_{5}$ is also shown, as well as the package to $\mathrm{PCB}$ transition. A 3D representation of the model is shown in Fig. 6.

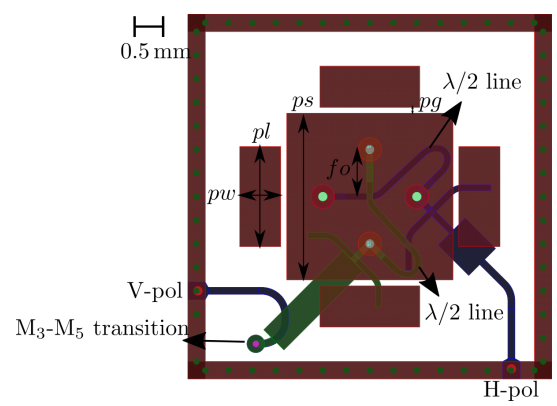

Fig. 5. Final layout with feeding and matching networks, inter layer and PCB to package transitions (ground planes and grounding vias omitted).

The full system was simulated using ADS Momentum and the magnitude of the S-parameters on V-pol (port 1) and H-pol (port 2) ports when fed from a $50 \Omega$ microstrip line on the host 


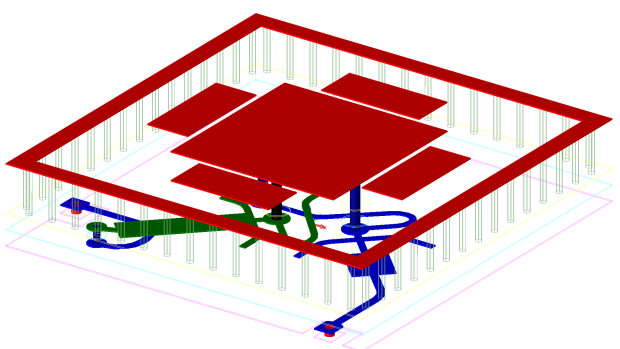

Fig. 6. 3D model of full layout (ground planes and grounding vias omitted).

PCB, are shown in Fig. 7, where it can be seen that not only the reflection coefficient magnitude is below $-10 \mathrm{~dB}$ in both ports, but also the isolation is greater than $40 \mathrm{~dB}$ over the bandwidth of $24.25 \mathrm{GHz}$ to $27.5 \mathrm{GHz}$. The E-Plane and H-Plane radiation patterns at the centre frequency of $26 \mathrm{GHz}$ are shown in Fig. 9. It can be seen that the radiation pattern suffers practically no change from the beginning to the end of the $5 \mathrm{G}$ Ka-band. Such is due to the differential feed topology which reinforces the fundamental $\mathrm{TM}_{100}$ and $\mathrm{TM}_{010}$ degenerate modes and cancels higher order modes. A radiation efficiency of approximately $60 \%$ and a peak gain of $5 \mathrm{dBi}$ was observed in both $\mathrm{V}$-pol and H-pol ports.

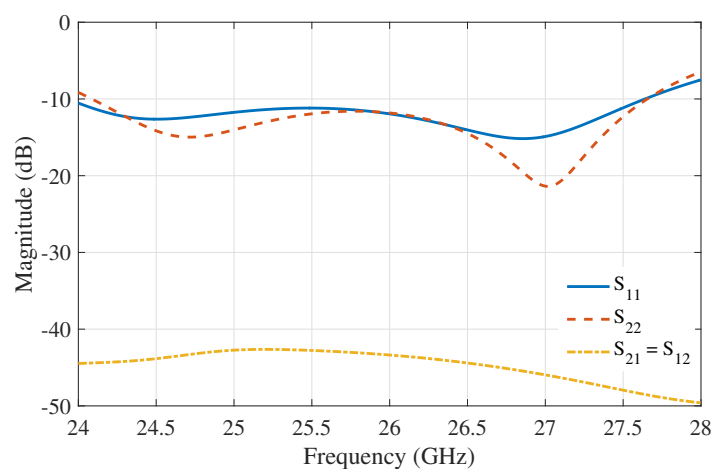

Fig. 7. Simulated results for the magnitude of V-Pol (port 1) and H-Pol (port 2) S-parameters.

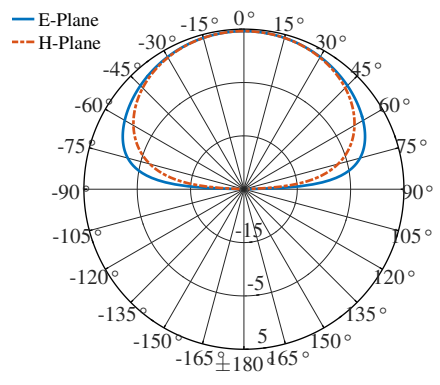

(a)

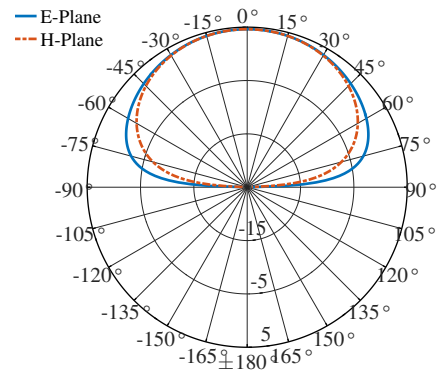

(b)
Fig. 8. Simulated V-pol (a) and H-pol (b) radiation patterns at $24.25 \mathrm{GHz}$.

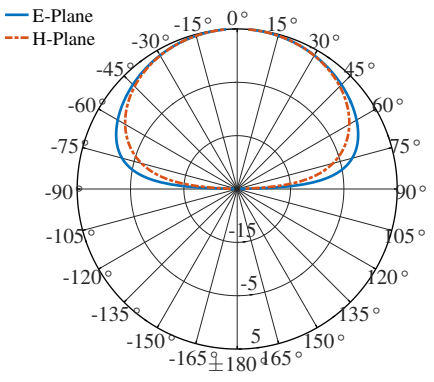

(a)

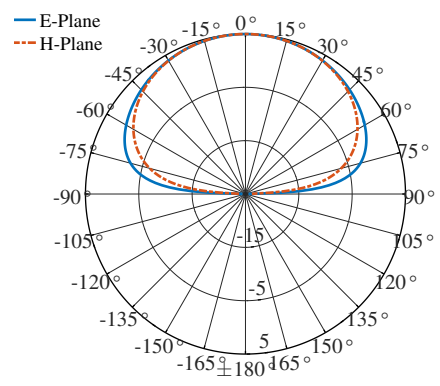

(b)
Fig. 9. Simulated V-pol (a) and H-pol (b) radiation patterns at $26 \mathrm{GHz}$.

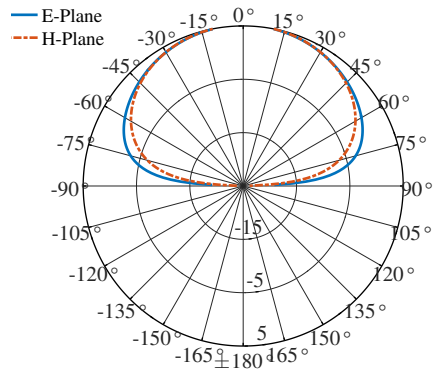

(a)

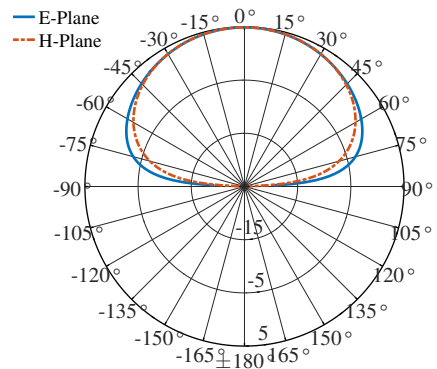

(b)
Fig. 10. Simulated V-pol (a) and H-pol (b) radiation patterns at $27.5 \mathrm{GHz}$.

\section{CONClusions}

In this work a new topology of patch antenna-in-package was shown. The simulation results showed a very high isolation and a good match in both ports. Nonetheless, despite reducing the number of layers for the antenna, extra complexity is added in the matching process. Furthermore, it was shown that with our design that a frequency stable radiation pattern, from $24.25 \mathrm{GHz}$ to $27.5 \mathrm{GHz}$, can be obtained.

In the future we are aiming at manufacturing and measuring the performance of the antenna and exploit techniques to reduce the matching network complexity.

\section{ACKNOWLEDGMENT}

This work was supported by FCT (Fundação para a Ciência e a Tecnologia, Portugal), through the Ph.D. grant PD/BD/128197/2016.

\section{REFERENCES}

[1] T. Tjelta, S. Temple, and R. W. Mohr, "Euro-5G-Supporting the European 5G Initiative," 2015.

[2] C. Balanis, Antenna Theory: Analysis and Design. Wiley, 2016

[3] C. A. Balanis, Modern antenna handbook. John Wiley \& Sons, 2011.

[4] G. Kumar and K. Ray, Broadband Microstrip Antennas, ser. Artech House antennas and propagation library. Artech House, 2003.

[5] H. Nawaz and I. Tekin, "Dual Polarized, Differential Fed Microstrip Patch Antennas with very High Inter-port Isolation for Full Duplex Communication," IEEE Transactions on Antennas and Propagation, vol. PP, no. 99, pp. 1-1, 2017. 